\title{
Exact solutions of a particle in a box with a delta function potential: The factorization method
}

\author{
Pouria Pedram* \\ Plasma Physics Research Center, Science and Research Campus, \\ Islamic Azad University, P.O. Box 14665-678, Tehran, Iran \\ M. Vahabi \\ Department of Physics, Shahid Beheshti University, G. C., Evin, Tehran 19839, Iran

\begin{abstract}
We use the factorization method to find the exact eigenvalues and eigenfunctions for a particle in a box with the delta function potential $V(x)=\lambda \delta\left(x-x_{0}\right)$. We show that the presence of the potential results in the discontinuity of the corresponding ladder operators. The presence of the delta function potential allows us to obtain the full spectrum in the first step of the factorization procedure even in the weak coupling limit $\lambda \rightarrow 0$.
\end{abstract}




\section{INTRODUCTION}

The time-independent form of Schrödinger's equation in the presence of a potential $V$ can be written as 1

$$
-\frac{\hbar^{2}}{2 m} \nabla^{2} \psi+V \psi=E \psi
$$

where $\psi$ is the wave function, $m$ is the mass of the particle, and $E$ is the eigenenergy. Equation (11) can be solved exactly only for a few potentials. A particle in a box and a

particle in a delta function potential are two well-known and instructive examples. $\underline{1}$ The former can be used to describe quantum dots and quantum wells at low temperatures, 2,3 and the latter can be used as a model for atoms and molecules. .4

The solution for a particle in a box with a delta function potential has been investigated using a perturbative expansion in the strength of the delta function potential $\lambda . \underline{5}$ Exact solutions have been obtained for the weak $(\lambda \rightarrow 0)$ and the strong $(1 / \lambda \rightarrow 0)$ coupling limits. $\underline{6}$

In this paper we discuss the solution for a particle in a box with a delta function potential using the factorization method and show that the presence of the delta function simplifies the factorization procedure. In this way we find the full spectrum of the Hamiltonian in the first step of the factorization method. We also show that this result applies in the weak coupling limit $\lambda \rightarrow 0$. Note that if we put $\lambda=0$ from the beginning, we need to continue the factorization procedure to find each eigenvalue in each step.

\section{PARTICLE IN A BOX WITH A DELTA FUNCTION POTENTIAL}

Consider a particle in a one-dimensional box of size $a$ with the delta function potential $V(x)=\lambda \delta\left(x-x_{0}\right)=\lambda \delta(x-p a)$, where $0<p<1$. In this case Eq. (11) takes the form

$$
-\frac{\hbar^{2}}{2 m} \frac{d^{2} \psi_{n}(x)}{d x^{2}}+\lambda \delta(x-p a) \psi_{n}(x)=E_{n} \psi_{n}(x)
$$

where $\psi_{n}(x)$ and $E_{n}$ are the corresponding eigenfunctions and eigenvalues, respectively. Because of the boundary conditions, $\psi_{n}(x)=0$ for $x \leq 0$ or $x \geq a$, the form of the eigenfunctions inside the box is

$$
\psi_{n}(x)= \begin{cases}A \sin \left(k_{n} x\right) & (0 \leq x \leq p a) \\ B \sin \left[k_{n}(x-a)\right] & (p a \leq x \leq a),\end{cases}
$$




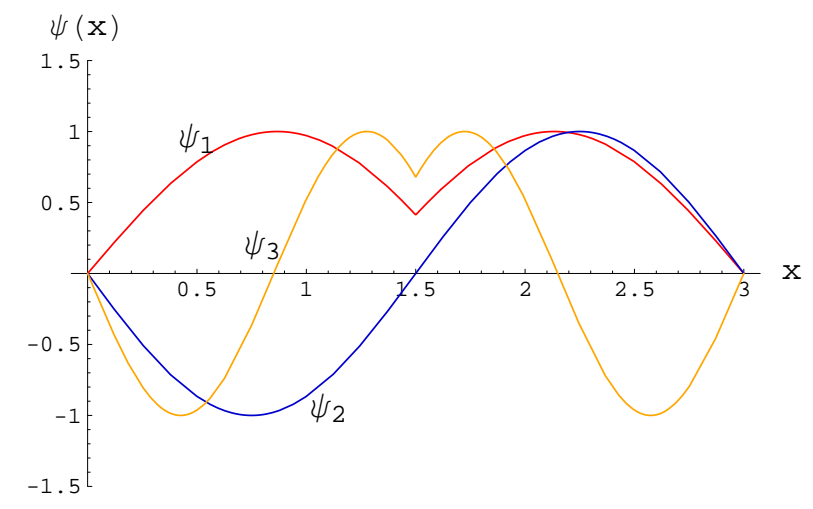

FIG. 1: First three stationary states $\psi_{n}(x)$ for $2 m \lambda / \hbar^{2}=8, a=3, A=1$, and $p=1 / 2$.

where $k_{n}=\sqrt{2 m E_{n}} / \hbar$. The continuity condition of the wave function at $x=p a$ gives $A / B=\sin \left[(p-1) k_{n} a\right] / \sin \left(p k_{n} a\right)$. Because the delta function is infinite at $x=p a$, the first derivative of the wave function is not continuous and the relation between the left and right derivatives of the wave function can be obtained by integrating Eq. (2) over the small interval $\left(x_{0}-\epsilon, x_{0}+\epsilon\right)$

$$
\begin{aligned}
\left.\frac{d \psi_{n}(x)}{d x}\right|_{p a+\epsilon}-\left.\frac{d \psi_{n}(x)}{d x}\right|_{p a-\epsilon} & =\frac{2 m}{\hbar^{2}} \int_{p a-\epsilon}^{p a+\epsilon} V(x) \psi_{n}(x) d x \\
& =\frac{2 m \lambda}{\hbar^{2}} \psi_{n}(p a) .
\end{aligned}
$$

We substitute the eigenfunctions in Eq. (3) in Eq. (4b) and obtain the quantization condition $\sqrt[5,6]{6}$

$$
k_{n} \sin \left(k_{n} a\right)=\frac{2 m \lambda}{\hbar^{2}} \sin \left(p k_{n} a\right) \sin \left[(p-1) k_{n} a\right] .
$$

The solutions to Eq. (5) give the energy spectrum of the Hamiltonian, $E_{n}=\hbar^{2} k_{n}^{2} / 2 m$. Figure 1 shows the first three stationary states for $2 m \lambda / \hbar^{2}=8, a=3, A=1$, and $p=1 / 2$.

\section{THE FACTORIZATION METHOD}

To calculate the eigenvalues and eigenfunctions of a Hamiltonian operator $H$, we can use a general operational procedure called the factorization method. In this method the Hamiltonian is written as the product of two ladder operators plus a constant, $H=a^{\dagger} a+E$. These operators are used to obtain the eigenfunctions of the Hamiltonian. In contrast to the simple harmonic oscillator, one ladder operator is usually not sufficient to form all the Hamiltonian's eigenfunctions and another ladder operator is needed for each eigenfunction. 
This method was first introduced by Schrödinger ${ }^{7-\underline{\underline{9}}}$ and Dirac $\underline{\underline{10}}$ and was further developed by Infeld and Hull 11 and Green. $\stackrel{12}{ }$ The spirit of the factorization method is to write the second-order differential operator $H$ as the product of two first-order differential operators $a$ and $a^{\dagger}$, plus a real constant $E$. The form of these operators depends on the form of the potential $V(x)$ and the factorization energy.

The procedure for finding the ladder operators and the eigenfunctions is as follows. $\stackrel{13}{ }$ We find operators $a_{1}, a_{2}, a_{3}, \ldots$ and real constants $E_{1}, E_{2}, E_{3}, \ldots$ from the recursive relations 13

$$
\begin{aligned}
& a_{1}^{\dagger} a_{1}+E_{1}=H, \\
& a_{2}^{\dagger} a_{2}+E_{2}=a_{1} a_{1}^{\dagger}+E_{1}, \\
& a_{3}^{\dagger} a_{3}+E_{3}=a_{2} a_{2}^{\dagger}+E_{2}, \ldots
\end{aligned}
$$

More generally

$$
a_{n+1}^{\dagger} a_{n+1}+E_{n+1}=a_{n} a_{n}^{\dagger}+E_{n}, \quad(n=1,2, \ldots) .
$$

Also assume that there exists a null eigenfunction (root function) $\left|\xi_{n}\right\rangle$ with zero eigenvalue for each $a_{n}$, namely,

$$
a_{n}\left|\xi_{n}\right\rangle=0
$$

Hence, $E_{n}$ is the $n$th eigenvalue of the Hamiltonian with the corresponding eigenfunction 13 (up to a normalization coefficient)

$$
\left|E_{n}\right\rangle=a_{1}^{\dagger} a_{2}^{\dagger} \ldots a_{n-1}^{\dagger}\left|\xi_{n}\right\rangle
$$

Figure 2 shows a schematic diagram describing the relation between root functions, the stationary states, and the ladder operators. As can be seen from Eq. (6), $a_{1}^{\dagger} a_{1}$ is equal to $H$ except for a constant. Thus, $a_{1}$ must have a linear momentum term to be consistent with the kinetic energy part of the Hamiltonian. According to Eq. (7), each of the annihilation operators $a_{n}$ should also have a a linear momentum term. Thus, $a_{n}$ can be written as

$$
a_{n}=\frac{1}{\sqrt{2 m}}\left[P+i f_{n}(x)\right]
$$

where $P$ is the momentum operator and $f_{n}(x)$ is a real function of $x$. Although these operators are not hermitian $\left(a_{n}^{\dagger}=\frac{1}{\sqrt{2 m}}\left(P-i f_{n}(x)\right) \neq a_{n}\right)$, their product is hermitian

$$
a_{n}^{\dagger} a_{n}=\frac{1}{2 m} P^{2}+\frac{1}{2 m} f_{n}^{2}+\frac{\hbar}{2 m} \frac{d f_{n}}{d x} .
$$




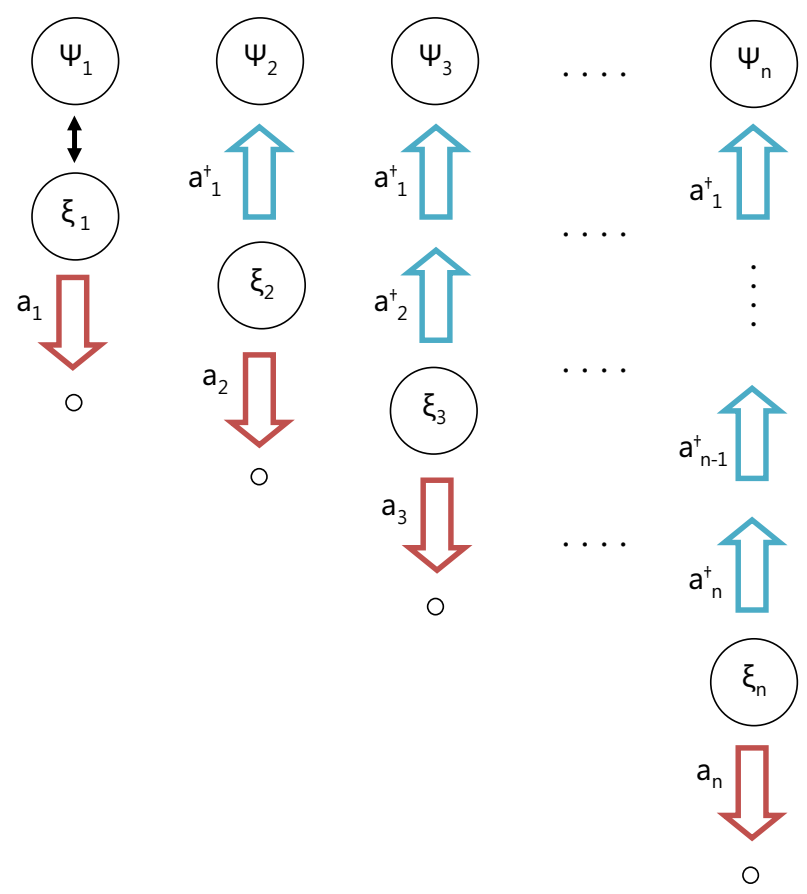

FIG. 2: A schematic diagram describing the relation between the root functions $\xi_{n}$, the stationary states $\Psi_{n}$, and the ladder operators $a_{n}, a_{n}^{\dagger}$.

We are now ready to find the ladder operators and eigenenergies for our problem. First, we consider Eq. (6) for $n=1$

$$
a_{1}^{\dagger} a_{1}+E_{1}=H
$$

From the form of the Hamiltonian (2) and Eq. (11) we can rewrite Eq. (12) as

$$
\frac{P^{2}}{2 m}+\frac{1}{2 m} f_{1}^{2}+\frac{\hbar}{2 m} \frac{d f_{1}}{d x}+E_{1}=\frac{P^{2}}{2 m}+\lambda \delta(x-p a)
$$

or equivalently

$$
\frac{1}{2 m} f_{1}^{2}+\frac{\hbar}{2 m} \frac{d f_{1}}{d x}+E_{1}=\lambda \delta(x-p a)
$$

Note that in contrast to Eq. (21), Eq. (14) is a nonlinear first-order differential equation.

To solve Eq. (14), we consider the left-hand and right-hand sides of the delta function potential separately. For these regions, Eq. (14) reduces to

$$
\frac{1}{2 m} f_{1}^{2}+\frac{\hbar}{2 m} \frac{d f_{1}}{d x}+E_{1}=0 \quad(x \neq p a)
$$

which has the solution

$$
f_{1}(x)=\sqrt{2 m E_{1}} \cot \left[\frac{\sqrt{2 m E_{1}}}{\hbar}(x-b)\right]
$$


where $b$ is a constant of integration. We require that $f_{1}(x)$ be finite in the range $0<x<a$, where it is the solution for our problem. Because the singularities of the cotangent function are $\pi$ radian apart, we choose the points $x=0$ and $x=a$ as the singularity points of $f_{1}(x)$. At these points the potential and hence the Hamiltonian are infinite and $f_{1}(x)$ can be infinite at the boundaries. Hence, we have

$$
f_{1}(x)= \begin{cases}\sqrt{2 m E_{1}} \cot \left[\frac{\sqrt{2 m E_{1}}}{\hbar} x\right], & (x<p a) \\ \sqrt{2 m E_{1}} \cot \left[\frac{\sqrt{2 m E_{1}}}{\hbar}(x-b)\right], & (x>p a),\end{cases}
$$

where $\left(\sqrt{2 m E_{1}} / \hbar\right)(a-b)=\pi$. To fix the value of $E_{1}$, we need to use the discontinuity relation of the ladder operators, which can be obtained by integrating Eq. (14) over a small interval $(p a-\epsilon, p a+\epsilon)$

$$
f_{1}(p a+\epsilon)-f_{1}(p a-\epsilon)=\frac{2 m \lambda}{\hbar} .
$$

Equation (18) shows that the presence of the delta function results in the discontinuity of $f_{1}(x)$ at $x=p a$. We use Eq. (17) and $b=a-\pi \hbar \sqrt{2 m E_{1}}$, to rewrite Eq. (18) as

$$
\sqrt{2 m E_{1}}\left\{\cot \left[\frac{\sqrt{2 m E_{1}}}{\hbar}\left(p a+\frac{\pi \hbar}{\sqrt{2 m E_{1}}}-a\right)\right]-\cot \left[\frac{\sqrt{2 m E_{1}}}{\hbar}(p a)\right]\right\}=\frac{2 m \lambda}{\hbar} .
$$

Because $\cot (\pi+\alpha)=\cot \alpha$, Eq. (19) can be written in the form

$$
k_{1}\left\{\cot \left[(p-1) k_{1} a\right]-\cot \left(p k_{1} a\right)\right\}=\frac{2 m \lambda}{\hbar^{2}},
$$

or

$$
k_{1} \sin \left(k_{1} a\right)=\frac{2 m \lambda}{\hbar^{2}} \sin \left(p k_{1} a\right) \sin \left[(p-1) k_{1} a\right]
$$

where $k_{1}=\sqrt{2 m E_{1}} / \hbar$. Equation (20) is similar to Eq. (5). Note that, although we calculated the ground state energy of the Hamiltonian in the first step of the factorization method, we found the full spectrum. This conclusion applies to the weak coupling limit $\lambda \rightarrow 0$. In this limit $\sin \left(k_{1} a\right)=0$ from Eq. (21), which gives the full spectrum of a particle in a box with $k_{1}=n \pi / a$. This result has an interesting consequence. If we use the factorization method to calculate the energy levels of a particle in a box with $\lambda=0$, we cannot find all of them in the first step of the procedure. In fact, we should use the recursive relations to find each

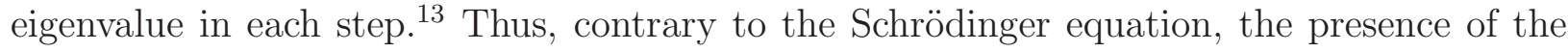
delta function in the factorization method simplifies the problem and repeated application of the recursion relations is not necessary. We need only to replace the index 1 with $n$ in Eqs. (17) and (21). 
To obtain the root functions, we rewrite Eq. (8) as

$$
\begin{array}{rlrl}
\left(\frac{\hbar}{i} \frac{d}{d x}+i \hbar k_{n} \cot \left(k_{n} x\right)\right) \xi_{n}(x) & =0, \quad & & (x<p a), \\
\left(\frac{\hbar}{i} \frac{d}{d x}+i \hbar k_{n} \cot \left[k_{n}(x-b)\right]\right) \xi_{n}(x) & =0, \quad(x>p a),
\end{array}
$$

where $\xi_{n}(x) \equiv\left\langle x \mid \xi_{n}\right\rangle$. It can be easily checked that the following is a solution to Eq. (22)

$$
\xi_{n}(x)= \begin{cases}A \sin \left(k_{n} x\right), & (x<p a), \\ -B \sin \left[k_{n}(x-b)\right], & (x>p a) .\end{cases}
$$

Because in the first step of the factorization procedure the root function $\xi_{1}(x)$ is equal to the first eigenfunction $\psi_{1}(x)$ (see Eq. (9) and Fig. 2) and we have replaced the index 1 by $n$, all the root functions are equal to the eigenfunctions of the Hamiltonian, that is, $\xi_{n}(x)=\psi_{n}(x)$. This result is similar to Eq. (3) for $x<p a$. Moreover, because $k_{n} b=k_{n} a-\pi$, we have $\sin \left[k_{n}(x-b)\right]=\sin \left[k_{n}(x-a)+\pi\right]=-\sin \left[k_{n}(x-a)\right]$. So, these eigenfunctions are equivalent to the solutions in Eq. (3).

\section{SUGGESTED PROBLEMS}

Problem 1. Use the recursive relations (6) to show that $\left|E_{n}\right\rangle$ in Eq. (9) is the eigenfunction of the Hamiltonian with $E_{n}$ as its eigenvalue.

Problem 2. Show that the eigenvalues $E_{1}, E_{2}, E_{3}, \ldots$ form a monotonic increasing sequence, that is, $E_{1} \leq E_{2} \leq E_{3} \leq \ldots$.

Problem 3. Apply the factorization method to a particle in a box with no delta function potential. In the first step show that $k_{1}=\pi / a$. Continue the factorization procedure to find all the eigenenergies and eigenfunctions. Hint: consider $a_{n}=\frac{1}{\sqrt{2 m}}\left[P+i c_{n} \cot \left(d_{n} x\right)\right]$, where $c_{n}$ and $d_{n}$ are real constants.

* Electronic address: pouria.pedram@gmail.com

1 D. J. Griffiths, Introduction to Quantum Mechanics (Prentice Hall, Upper Saddle River, NJ, 2004).

2 B. I. Shklovskii and A. L. Efros, Electronic Properties of Doped Semiconductors (Springer, New York, 1984), Chap. 1. 
3 P. Y. Yu and M. Cardona, Fundamentals of Semiconductors (Springer, New York, 2005), Chap. 9.

4 I. R. Lapidus, "Relativistic one-dimensional hydrogen atom," Am. J. Phys. 51, 1036-1038 (1983).

5 N. Bera, K. Bhattacharya, and J. K. Bhattacharjee, "Perturbative and non-perturbative studies with the delta function potential," Am. J. Phys. 76, 250-257 (2008).

6 Y. N. Joglekara, "Particle in a box with a $\delta$-function potential: Strong and weak coupling limits," Am. J. Phys. 77, 734-736 (2009).

7 E. Schrödinger, "A method of determining quantum-mechanical eigenvalues and eigenfunctions," Proc. Roy. Irish Acad. Sect. A 46, 9-16 (1941).

8 E. Schrödinger, "Further studies on solving eigenvalue problems by factorization," Proc. Roy. Irish Acad. Sect. A 46, 183-206 (1941).

9 E. Schrödinger, "The factorization of the hypergeometric equation," Proc. Roy. Irish Acad. Sect. A 47, 53-54 (1941) or arXiv:physics/9910003.

10 P. M. Dirac, The Principles of Quantum Mechanics (Oxford University Press, Oxford, 1958).

11 I. Infeld and T. E. Hull, "The factorization method," Rev. Mod. Phys. 23, 21-68 (1951).

12 H. S. Green, Matrix Methods in Quantum Mechanics (Barnes \& Noble, New York, 1968).

13 H. C. Ohanian, Principles of Quantum Mechanics (Prentice Hall, Englewood Cliffs, NJ, 1990). 